\title{
Effect of variable thermal conductivity on entropy generation in a plate with internal energy generation
}

\author{
T. K. Favas $^{1 *}$ and G. Jilani ${ }^{1}$ \\ ${ }^{1}$ Department of Mechanical Engineering, National Institute of Technology Calicut, Kerala, India
}

\begin{abstract}
The current numerical investigation aims at analyzing the effect of variable thermal conductivity on local and global entropy generation rates in an energy generating plate dissipating heat by conjugate conduction-forced convection heat transfer. In order to fulfill this objective, the physical model of the plate dissipating heat into surrounding coolant is transformed into a mathematical model governing the temperature field in the plate as well as flow and thermal fields in the fluid. The resulting mathematical model, being a set of coupled and non linear partial differential equations, is solved by adopting stream functionvorticity formulation and by employing Alternating direction implicit scheme. Keeping Prandtl number of the fluid, temperature of the free stream coolant and maximum permissible plate temperature as fixed, numerical predictions are obtained for wide range of values of aspect ratio, conduction-convection parameter, energy generation parameter and flow Reynolds number. It is concluded that unrealistic constant thermal conductivity assumption leads to underestimation of entropy generation rates. It is also found that an increase in energy generation parameter results in significant increase in underestimation of global entropy generation rate.
\end{abstract}

\section{Introduction}

Plates with volumetric energy source find application in nuclear reactors [1], printed circuit boards [2], thermal insulation, metal casting, heat transfer gauges [3], hardening of structural materials [4], wall heating systems [5], laser irradiation [6], blade shaped electrical resistance heater [7], curing of concrete slabs [8] etc. For example, in printed circuit boards and packages, the highest temperature called as 'hot spot' must not exceed a specified ceiling value. If the temperature rises above this ceiling value, the error-free operation of the electronic device is threatened and hence heat dissipation to the surrounding cooling medium is highly essential [9]. Nuclear reactor is another application where the energy generated as a result of fission process in a nuclear fuel element is dissipated to surrounding stream of coolant by combined conduction-convection process so as to prevent from detrimental changes in the fuel element material by maintaining the

\footnotetext{
*Corresponding author: favastk@gmail.com
} 
maximum temperature well below certain limit [10]. These heat transfers, occurring in electronic boards and nuclear fuel elements, through finite temperature difference will definitely lead to entropy generation [11]. As entropy generation is a measure of lost available work [11], it has to be minimized for an optimum design of the concerned thermal system. Due to the preceding facts, a number of investigators have paid their attention to studies on entropy generation in energy generating plates in the recent past. A brief review of the literature which is closely related to the present study is illustrated below.

Ruocco [12] numerically predicted entropy generation rates in a plate mounted with discrete energy sources dissipating heat into a laminar impinging jet. Shuja et al. [13] numerically predicted entropy generation rates arising out of cooling of an energy generating rectangular block having constant thermal conductivity. Ibanez et al. [14] analytically predicted entropy generation rates associated with unidirectional conduction in an energy generating plate with constant thermal conductivity by imposing average convective heat transfer co-efficient over its surfaces. Bautista et al. [15] analytically studied the problem of entropy generation associated with unsteady state unidirectional conduction in a constant thermal conductivity slab having uniform internal energy source. El Haj Assad [6] analytically predicted entropy generation rates associated with unidirectional conduction in a non-uniformly energy generating slab with constant thermal conductivity by imposing average heat transfer co-efficient over its surfaces. Aziz and Khan [16] analytically and numerically investigated the problem of entropy generation associated with steady unidirectional conduction in an energy generating plate by considering dependence of thermal conductivity on temperature. Sahin [17] analytically studied the problem of entropy generation arising out of heat transfer by conduction in a wall. Assuming uniform wall temperature boundary conditions, the consequences of variable thermal conductivity and internal energy source on global entropy generation rate were investigated. Aziz and Khan [18] analytically obtained entropy generation rates in an energy generating slab having constant thermal conductivity by assuming steady onedimensional conduction within the slab and average heat transfer co-efficient over its surfaces. Torabi and Zhang [19] analytically investigated the problem of entropy generation associated with conduction heat transfer in a plane wall with variable thermal conductivity and internal energy source by assuming convective along with radiative conditions over the heat dissipating surfaces.

The review of the literature pertinent to entropy generation studies in plates with volumetric energy sources undoubtedly discloses that except Aziz and Khan [16], Sahin [17] and Torabi and Zhang [19], no one has paid their concentration to the study of entropy generation associated with cooling of a plate with volumetric energy source by taking into consideration of variation of thermal conductivity with temperature of the plate. Nonetheless, these studies are dealing with idealistic assumption of unidirectional conduction within the plate. Besides, idealistic situations of either uniform wall temperature or uniform convective heat transfer co-efficient are imposed on the heat dissipating surfaces. The shortfalls of the earlier studies are the inspiration for the current study. Thus, the main objective of this study is analyzing the outcome of thermal conductivity variation with temperature on local and global entropy generation rates in an energy generating plate by considering more realistic two-dimensional conjugate conduction-forced convection heat transfer analysis.

\section{Mathematical formulation}

Fig. 1 depicts a plate with uniform volumetric energy generation having dimensions $H$ and $2 W$ dissipating heat into an upcoming stream of coolant having free stream velocity and temperature $U_{\infty}$ and $T_{\infty}$ respectively. The Prandtl number of the coolant is $\operatorname{Pr}$. The energy 
generated within the plate is first transferred to its heat dissipating surfaces by heat conduction process resulting in entropy generation and finally dissipated by forced convection to the surrounding fluid stream. Fig. 2 illustrates the computational model with respect to the physical domain explained above. Obviously, temperature distribution within the plate and flow and thermal fields within the fluid will be symmetric about the centre line of the plate. Thus, in the present study, the right half of the physical domain is taken as the computational domain as depicted in Fig. 2.

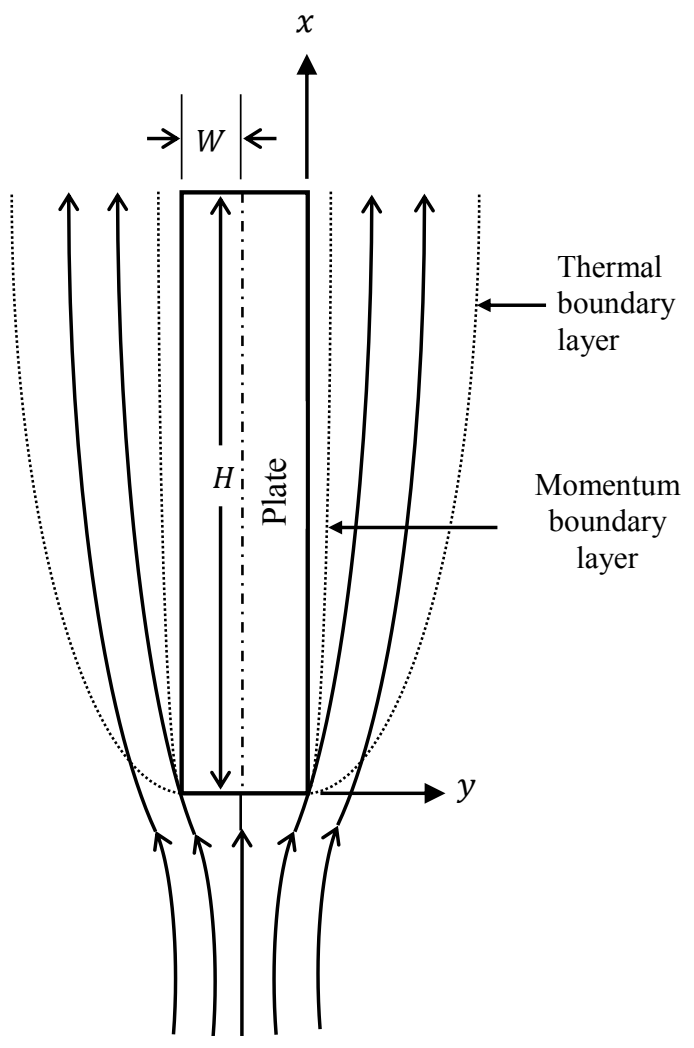

Fluid stream at $U_{\infty}, T_{\infty}$

Fig. 1. Physical model.

With the intention of numerically simulating the problem depicted above, the following supplementary assumptions are introduced for obtaining the most appropriate mathematical model.

(i) The plate material is isotropic and homogenous.

(ii) The heat conduction in the plate is two-dimensional.

(iii) The flow of the coolant is incompressible, laminar, and two-dimensional.

(iv) Coolant is viscous and Newtonian.

(v) Effect of viscous dissipation is negligibly small.

(vi) The effect of thermal radiation on heat dissipation rate is negligible.

Taking into account the assumptions stated, dimensionless equations governing temperature field in the plate as well as thermal and flow fields in the fluid domain are deduced as follows: 


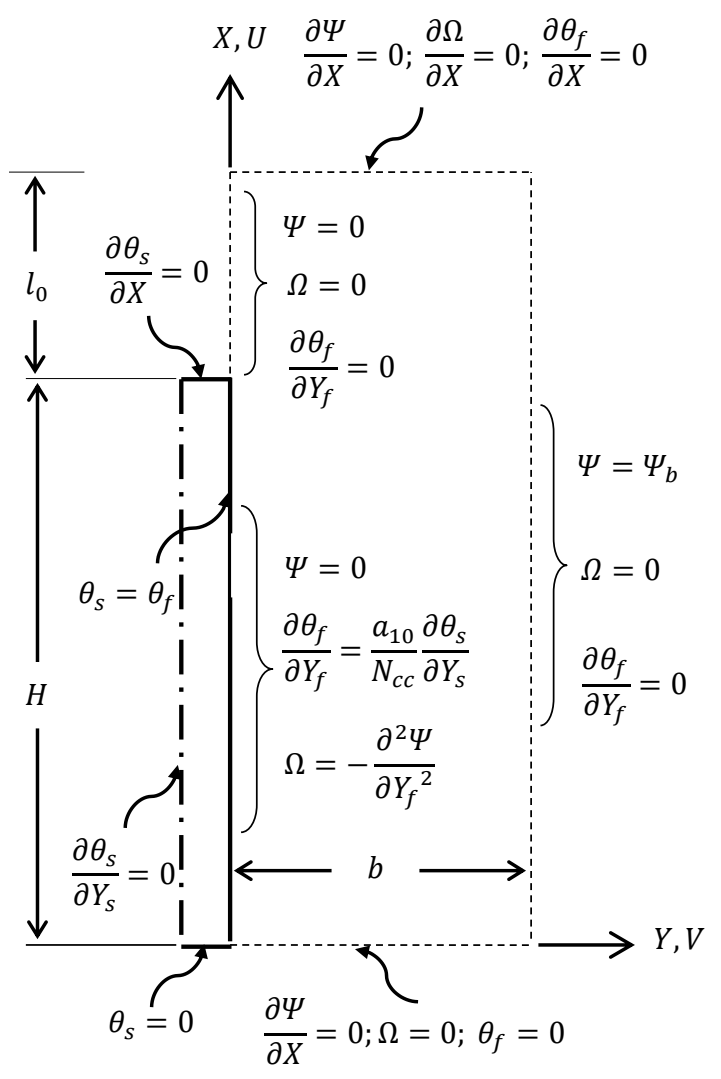

Fig. 2. Computational domain.

Solid domain:

$\frac{\partial^{2} \theta}{\partial X^{2}}+C \frac{\partial^{2} \theta}{\partial Y_{s}^{2}}-\frac{a_{3}}{\left(a_{4}+a_{3} \theta\right)}\left(\frac{\partial \theta}{\partial X}\right)^{2}-\frac{a_{5}}{\left(a_{4}+a_{3} \theta\right)}\left(\frac{\partial \theta}{\partial Y_{s}}\right)^{2}+\left(a_{6} \theta+C\right) Q=0$

Where, $\theta$ is the dimensionless temperature in the plate and $Q$ is the energy generation parameter. The constants in Eqn. (1) are defined as

$C=4 A_{r}^{2} \quad a_{3}=a_{2}\left(T_{0}-T_{\infty}\right) \quad a_{4}=a_{1}+a_{2} T_{\infty} \quad a_{5}=C a_{3} \quad a_{6}=\frac{a_{5}}{a_{4}}$

Where, $A_{r}$ is the plate aspect ratio and $T_{0}$ is maximum allowable temperature in the plate. The presence of constants $a_{1}$ and $a_{2}$ in the expressions of the constants $a_{3}$ and $a_{4}$ is due to dependence of thermal conductivity $k_{s}$ on plate temperature $T_{s}$ which is expressed for Uranium Dioxide, a commonly used nuclear fuel element material as [10].

$k_{s}=\frac{1}{a_{1}+a_{2} T_{s}}$

Eqn. (1) is a highly non-linear partial differential equation and therefore it has to be linearized before seeking its numerical solution. The linearized form of this equation can be obtained as

$\frac{\partial^{2} \theta}{\partial X^{2}}+C \frac{\partial^{2} \theta}{\partial Y_{s}^{2}}-a_{7} \frac{\partial \theta}{\partial X}-a_{8} \frac{\partial \theta}{\partial Y_{s}}+a_{9} Q=0$

Where, 
$a_{7}=\frac{a_{3}}{\left(a_{4}+a_{3} \theta\right)}\left(\frac{\partial \theta}{\partial X}\right) \quad a_{8}=\frac{a_{5}}{\left(a_{4}+a_{3} \theta\right)}\left(\frac{\partial \theta}{\partial Y_{S}}\right) \quad a_{9}=a_{6} \theta+C$

Fluid domain:

$\frac{\partial^{2} \Psi}{\partial X^{2}}+\frac{\partial^{2} \Psi}{\partial Y_{f}^{2}}=-\Omega$

$U \frac{\partial \Omega}{\partial X}+V \frac{\partial \Omega}{\partial Y_{f}}=\frac{1}{R e_{H}}\left(\frac{\partial^{2} \Omega}{\partial X^{2}}+\frac{\partial^{2} \Omega}{\partial Y_{f}^{2}}\right)$

$U \frac{\partial \theta}{\partial X}+V \frac{\partial \theta}{\partial Y_{f}}=\frac{1}{R e_{H} \operatorname{Pr}}\left(\frac{\partial^{2} \theta}{\partial X^{2}}+\frac{\partial^{2} \theta}{\partial Y_{f}^{2}}\right)$

Where, $\Psi$ and $\Omega$ represents dimensionless stream function and vorticity respectively.

Once plate temperature field is found, the dimensionless local and global entropy generation rates $S_{l}$ and $S_{g}$ can be computed using the following equations:

$S_{l}=\frac{a_{10}}{\left(\theta+\theta_{\infty}\right)^{2}}\left(\left(\frac{\partial \theta}{\partial Y_{S}}\right)^{2}+\frac{1}{C}\left(\frac{\partial \theta}{\partial X}\right)^{2}\right)$

$S_{g}=2 \int_{0}^{-1} \int_{0}^{1} S_{l} d X d Y_{S}$

Where,

$$
a_{10}=\frac{a_{4}}{\left(a_{4}+a_{3} \theta\right)}
$$

The dimensionless parameters and variables used in this work are expressed as

$$
\begin{aligned}
& X=\frac{x}{H} \quad Y_{s}=\frac{y}{W} \quad Y_{f}=\frac{y}{H} \quad U=\frac{u}{U_{\infty}} \quad V=\frac{v}{U_{\infty}} \quad \theta=\frac{T-T_{\infty}}{T_{0}-T_{\infty}} \\
& A_{r}=\frac{H}{2 W} \quad N_{c c}=\frac{k_{f}}{k_{s \infty}}\left(\frac{W}{H}\right) \quad P r=\frac{\mu_{f} c_{p}}{k_{f}} \quad Q=\frac{q^{\prime \prime \prime} W^{2}}{k_{s \infty}\left(T_{0}-T_{\infty}\right)} \\
& \operatorname{Re}_{H}=\frac{\rho_{f} U_{\infty} H}{\mu_{f}} \quad S_{l}=\frac{S_{g e n}^{\prime \prime \prime} W^{2}}{k_{S \infty}} \quad S_{g}=\frac{S_{g e n} W}{k_{s \infty} H} \quad \theta_{\infty}=\frac{T_{\infty}}{T_{0}-T_{\infty}}
\end{aligned}
$$

\section{Numerical solution}

Eqns. (3) - (6) are coupled partial differential equations of non-linear nature and therefore, these equations have to be solved numerically in an iterative manner. While Eqns. (3) and (4) are discretized using central difference schemes and obtained algebraic equations are solved numerically by employing Line-by-Line Gauss-Seidel iterative method, Eqns. (5) and (6) in their pseudo-transient form are discretized using ADI scheme and obtained algebraic equations are solved iteratively by employing Thomas Algorithm. Once the converged values of plate temperature is obtained, local and global rates of entropy generation are computed by means of Eqn. (7) and Eqn. (8), respectively.

\section{Results and discussion}

The current numerical investigation deals with the outcome of variable thermal conductivity $k_{s}$ on entropy generation arising out of conjugate conduction-forced convection in a plate having uniform volumetric heat sources. Keeping maximum 
permissible plate temperature $T_{0}$, temperature of the free stream coolant $T_{\infty}$ and Prandtl number of the fluid $\mathrm{Pr}$ as constant at $2354 \mathrm{~K}, 673 \mathrm{~K}$ and 0.005 respectively, results are exhibited in the form of transverse and axial local entropy generation rate $S_{l}$ profiles and variation of global entropy generation rate $S_{g}$ with plate aspect ratio $A_{r}$, conductionconvection parameter $N_{c c}$, flow Reynolds number $R e_{H}$ and energy generation parameter $Q$ for temperature dependent and temperature independent thermal conductivity cases.

Fig. 3 depicts the consequence of variable $k_{S}$ on transverse $S_{l}$ profiles in the plate while keeping $A_{r}=10, N_{c c}=0.50, Q=0.50$ and $R e_{H}=2500$ as fixed. It is very much clear from this figure that $S_{l}$ takes its minimum value at $Y_{S}=-1$, and it keeps on increasing to its maximum value at $Y_{S}=0$, for both constant and variable thermal conductivity cases. It is also evident that constant thermal conductivity assumption results in significant underestimation of $S_{l}$ except in the region close to the centre line of the plate. Further, it is worth noticing that an underestimation of $31.41 \%$ in $S_{l}$ can be noticed at $Y_{s}=0$, i.e, along the solid-fluid interface.

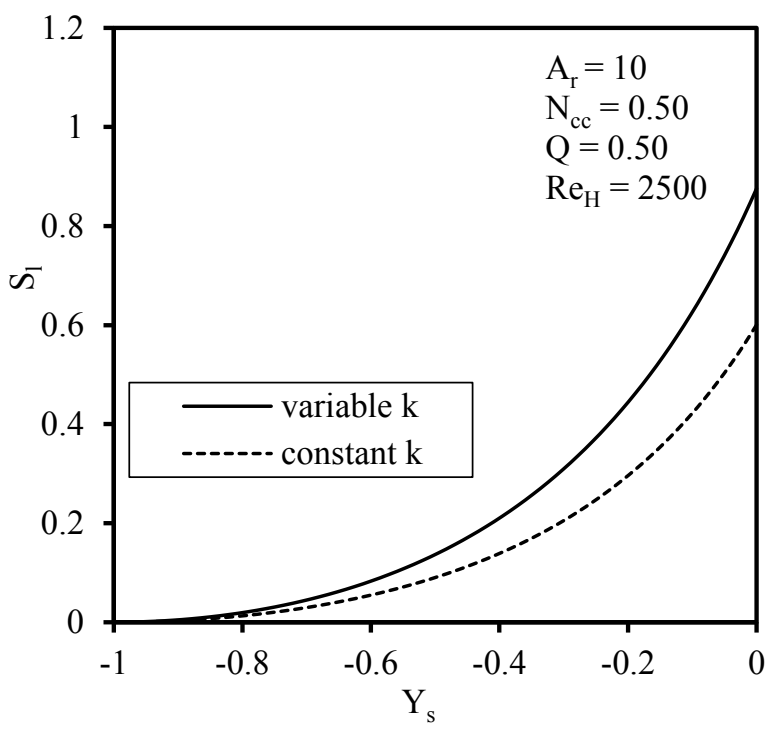

Fig. 3. Transverse $S_{l}$ profiles for constant and variable $k_{S}$ studies at $X=0.50$

Fig. 4 presents the result of variable $k_{s}$ on $S_{l}$ profile along solid-fluid interface while keeping the values of $A_{r}=10, N_{c c}=0.50, Q=0.50$ and $R e_{H}=2500$ as constant. It is clearly evident that $S_{l}$ takes its minimum value near the leading edge of the plate, it keeps on increasing abruptly to maximum value at $X=0.125$, and thereafter gradually diminishes towards the trailing edge. Further, as discussed in Fig. 3, it is very much evident that the idealistic constant thermal conductivity assumption results in underestimation of $S_{l}$. Furthermore, it is worth noticing that error in prediction of $S_{l}$ increases to maximum value near trailing edge of the plate. Precisely, error in prediction of $S_{l}$ is as high as $38.17 \%$ at $X$ $=1$, which represents the trailing edge of the plate.

Fig. 5 exhibits the consequence of variable thermal conductivity on the variation of $S_{g}$ in the plate with aspect ratio $A_{r}$, while $N_{c c}, Q$ and $R e_{H}$ are kept constant. It is abundantly clear that for constant and variable $k_{s}$, the value of $S_{g}$ is almost invariant with $A_{r}$. It can be also noticed that constant thermal conductivity assumption results in considerable underestimation of $S_{g}$. Accurately, error in calculation of $S_{g}$ increases from $28.29 \%$ to $30.49 \%$ as $A_{r}$ varies from 5 to 15 . 


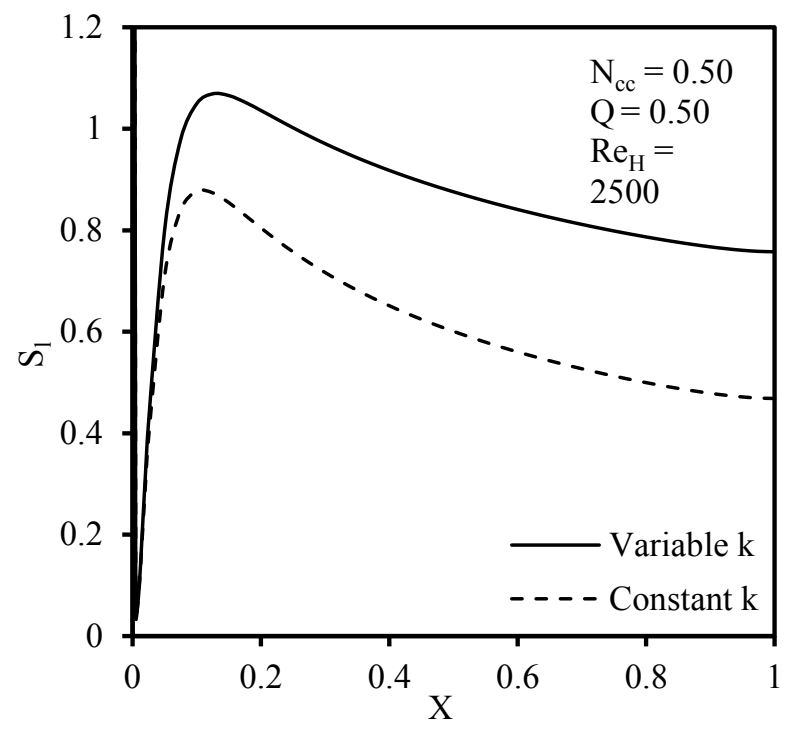

Fig. 4. Axial $S_{l}$ profiles at solid-fluid interface for temperature dependent and constant $k_{s}$ studies

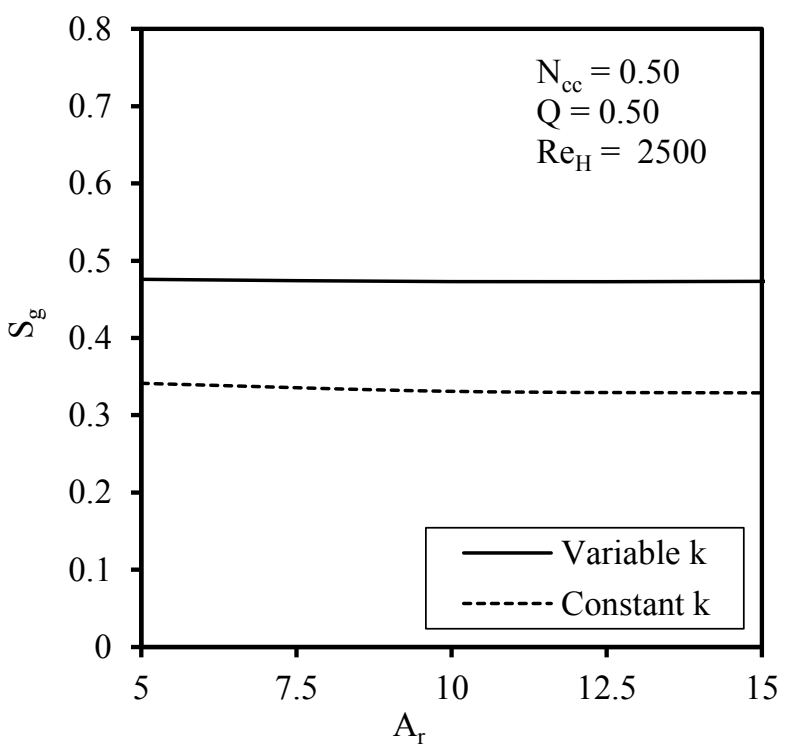

Fig. 5. Variation of $S_{g}$ with $A_{r}$ for both variable and constant $k_{s}$ studies

Fig. 6 depicts the outcome of temperature dependent $k_{s}$ on the variation of $S_{g}$ with $N_{c c}$ while other parameters are being kept fixed. It is exclusively evident that for constant and variable $k_{s}, S_{g}$ increases considerably as $N_{c c}$ increases. Further, it is noticeable from this figure that $S_{g}$ increases almost in the same manner for both cases. Furthermore, it is important to emphasize here that the idealistic constant $k_{s}$ assumption results in erronious prediction of $S_{g}$ which remains almost consistent with increase in $N_{c c}$, but the percentage error diminishes from $32.19 \%$ to $25.97 \%$ as $N_{c c}$ varies from 0.40 to 0.70 


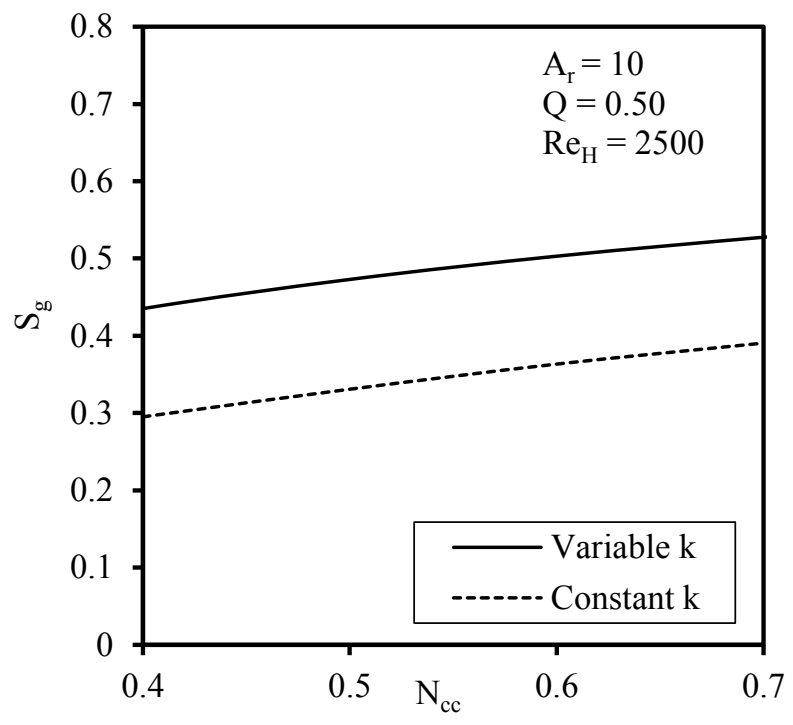

Fig. 6. Variation of $S_{g}$ with $N_{c c}$ for both constant and variable $k_{s}$ studies

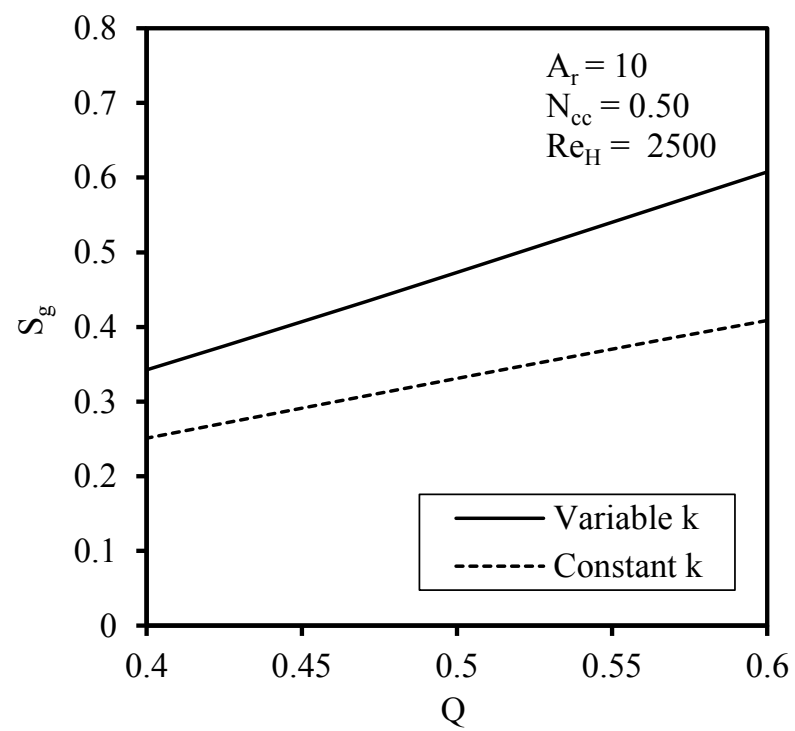

Fig.7. Variation of $S_{g}$ with $Q$ for both constant and variable $k_{s}$ studies

Fig. 7 illustrates the result of temperature dependent $k_{s}$ on the variation of $S_{g}$ with $Q$ for fixed values of $A_{r}, N_{c c}$ and $R e_{H}$. It is clear from this figure that for both cases, $S_{g}$ increases significantly with increase in $Q$. It is also evident that the rate of increase in $S_{g}$ with $Q$ is higher to some extent for variable $k_{s}$ case. Further, it is apparent that error in calculation of $S_{g}$ resulting from the idealistic constant $k_{s}$ assumption amplifies extensively while $Q$ takes its larger values. Furthermore, the under prediction in $S_{g}$ varies from $26.71 \%$ to $32.74 \%$ as $Q$ varies from 0.35 to 0.60 . 
Fig. 8 shows the result of temperature dependent $k_{s}$ on the variation of $S_{g}$ with $R e_{H}$ for fixed values of $A_{r}, N_{c c}$ and $Q$. It is quite visible that for constant and variable $k_{s}$, a raise in $R e_{H}$ results in considerable raise in $S_{g}$. It is also clear that the rate of increase in $S_{g}$ with $R e_{H}$ remains more or less same for both cases. Further, it is noticeable that the disparity in prediction of $S_{g}$ resulting from constant $k_{S}$ assumption remains more or less same with increase in $R e_{H}$, but the percentage error decreases from $31.70 \%$ to $27.92 \%$ as $R e_{H}$ increases from 1500 to 3500 .

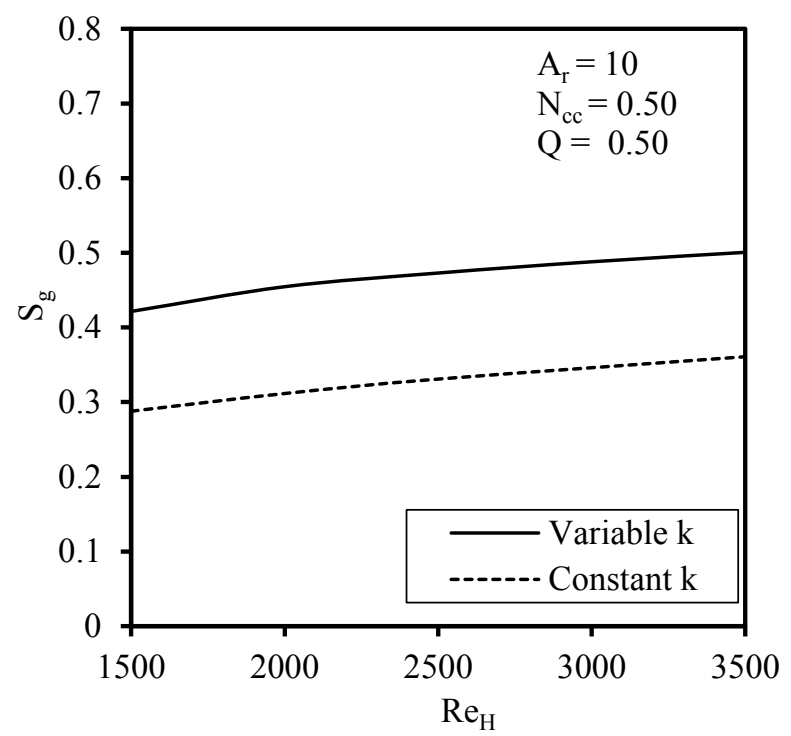

Fig. 8. Variation of $S_{g}$ with $R e_{H}$ for both constant and temperature dependent $k_{S}$ studies

\section{Conclusions}

The foremost aim of the current investigation is to inspect the consequence of thermal conductivity variation with temperature on local and global rates of entropy generation in a plate with volumetric energy source being cooled by forced convection. Employing finite difference method, the partial differential equations governing the plate temperature field, fluid flow and coolant thermal fields are solved at the same time in an iterative manner. Keeping Prandtl number of the fluid, temperature of the free stream coolant and maximum permissible plate temperature as fixed, results are expressed in terms of local and global entropy generation rates for different values of plate aspect ratio, conduction-convection parameter, flow Reynolds number and energy generation parameter. It is concluded that unrealistic constant thermal conductivity assumption in the plate results in significant under prediction of entropy generation rates. Further, it is concluded that an increase in energy generation parameter leads to significant increase in the underestimation of global entropy generation rate.

\section{References}

1. G. Kim, D.C. Davis, Nucl. Eng. Des. 158, 1-17 (1995)

2. S.M. Sawant, C. Gururaja Rao, Heat. Mass. Transf. 44, 1485-1495 (2008) 
3. A. Aziz, O.D. Makinde, J. Thermophys. Heat. Tr. 24(2), 438-444 (2010)

4. O.D. Makinde, A. Aziz, Heat. Mass. Transf. 47, 1407-1415 (2011)

5. M. Torabi, K. Zhang, Energ. Convers. Manage. 89, 12-23 (2015)

6. M. El Haj Assad, Int. J. Exergy. 9(3), 355-369 (2011)

7. A. Bejan, A.M. Morega, S.W. Lee, S.J. Kim, Int. J. Heat. Fluid. Fl. 14(2), 170-176 (1993)

8. A.J. Chapman, Heat Transfer (Macmillan Publishing Company, 1989)

9. S.J. Kim, S.W. Lee, Air Cooling Technology for Electronic Equipment (CRC Press, 1996)

10. R.H.S. Winterton, Thermal Design of Nuclear Reactors (Pergamon Press, 1981)

11. A. Bejan, Entropy generation through heat and fluid flow (John Wiley \& Sons, 1982)

12. G. Ruocco, Int. Commun. Heat. Mass. Transf. 24(2), 201-210 (1997)

13. S.Z. Shuja, B.S. Yilbas, M.O. Budair, I.S. Hussaini, Int. J. Energy Res. 23, 1133-1142 (1999)

14. G. Ibanez, S. Cuevas, M. Lopez de Haro, Int. J. Heat. Mass. Transf. 46, 1321-1328 (2003)

15. O. Bautista, F. Mendez, J.L. Martinez-Meyer, Int. J. Therm. Sci. 44, 570-576 (2005)

16. A. Aziz, W.A. Khan, Energy. 36, 6195-6207 (2011)

17. A.Z. Sahin, Int. J. Phys. Sci. 6(12), 2826-2831 (2011)

18. A. Aziz, W.A. Khan, Heat. Transf. Asian. Res. 4, 260-271 (2012)

19. M. Torabi, K. Zhang, Energy. 65, 387-397 (2014) 